\title{
Unhappiness and mortality: evidence from a middle-income Southeast Asian setting
}

\author{
Vasoontara Yiengprugsawan ${ }^{1 *}$, Sam-ang Seubsman ${ }^{1,2}$ and Adrian C Sleigh ${ }^{1}$
}

\begin{abstract}
Background: A relationship between happiness and mortality might seem obvious, but outside of affluent settings in developed countries there is almost no actual evidence that this is so.

Findings: We report our findings on happiness and mortality in Buddhist Southeast Asia. Our data are derived from a prospective nationwide cohort study of 60,569 Thai adults reporting in 2009 and followed up for all-cause mortality over the next four years (296 deaths). We also gathered data on a wide array of covariates and included these in the final model of the unhappiness-mortality effect. All final effect estimates were mutually adjusted odds ratios (AOR) and cohort members who reported being happy 'little' or 'none of the time' in 2009 were more likely to die (AOR 2.60, 95\% Confidence Interval 1.17-5.80). Other significant covariates include being female ( $<40$ years AOR 0.66, $\geq 40$ years AOR 0.57), unmarried (AOR 1.64) and current smokers (AOR 2.45).
\end{abstract}

Conclusion: Our study provides empirical evidence that the epidemiological effect of happiness is not confined to affluent Western countries, but it also increases the probability of staying alive in a middle-income Asian country.

Keywords: Happiness, Mortality, Psychological wellbeing, Cohort study, Thailand

\section{Findings}

When the $14^{\text {th }}$ Dalai Lama accepted his Nobel Prize in 1989 , he observed that "...we are all basically the same... we all seek happiness and try to avoid suffering." These connections were recognised in 1972 when King Jigme Wangchuck of Bhutan, drawing on Buddhist principles, famously focused development goals on boosting the Gross National Happiness [1]. A high level of happiness and minimal suffering has shown to interact to produce health and wellbeing [2-4] and Western studies report that subjective wellbeing, life satisfaction, and happiness not only associate with good health $[5,6]$ but also protect against premature mortality [7-9]. However, almost nothing has been reported about happiness effects on mortality in other economically poorer or culturally different (non-Western) settings.

In the field of positive psychology, happiness has been investigated from both subjective (hedonic) and psychological

\footnotetext{
*Correspondence: vasoontara.yieng@anu.edu.au

${ }^{1}$ National Centre for Epidemiology and Population Health, College of

Medicine, Biology and Environment, Research School of Population Health,

The Australian National University, Building 62, Mills Rd, Canberra, Acton

2601, Australia

Full list of author information is available at the end of the article
}

(eudaimonic) perspectives $[4,10]$. For quantitative population health research, self-reported happiness is commonly measured on scales including the World Values Survey and European Values Study, which have been systematically carried out between 1981 and 2007 [11]. Happiness was assessed by asking respondents to indicate how happy they were using four ordinal categories: very happy, rather happy, not very happy, and not at all happy. Subsequent studies on happiness have applied similar measures $[8,12,13]$.

We report our study of happiness and mortality in a Buddhist middle-income Southeast Asian country, using a large prospective cohort studying the health-risk transition among 87151 Thai adults. We began this cohort in 2005 with a comprehensive mail-out questionnaire to Open University distance-learning adults residing nationwide. The mean age was 29 years, half were female, half were urban, and most had modest incomes similar to the general Thai population $[14,15]$. In addition to a wide array of social and health information, we also collected Citizen IDs which were later used to match with national death records.

At the 2009 cohort follow-up $(n=60569)$, we measured happiness by self-report (five ordinal categories: 
Table 1 Unhappiness and mortality by cohort attributes, Thai Cohort Study

\begin{tabular}{|c|c|c|c|c|}
\hline \multirow[t]{2}{*}{ Cohort attributes } & \multirow{2}{*}{$\begin{array}{c}\% \text { proportion } \\
(\mathrm{n}=60569)\end{array}$} & \multicolumn{2}{|c|}{ \% reported unhappy* } & \multirow{2}{*}{$\begin{array}{c}\text { Bivariate odds ratio** } \\
{[95 \% \text { confidence intervals] }} \\
\text { for unhappiness by cohort attributes }\end{array}$} \\
\hline & & Alive in 2012 & Dead $(n=296)$ & \\
\hline Overall cohort in 2009 & & $5.2(3068)$ & $8.1(24)$ & \\
\hline \multicolumn{5}{|c|}{ Sociodemographic attributes } \\
\hline \multicolumn{5}{|l|}{ Age-sex groups: } \\
\hline Males $<40$ years & 24.8 & $5.3(1107)$ & $7.6(9)$ & Reference \\
\hline Females $<40$ years & 33.9 & $5.3(1554)$ & $10.5(8)$ & $0.95[0.86-1.05]$ \\
\hline Males $\geq 40$ years & 20.6 & $4.0(226)$ & $6.3(5)$ & $0.68[0.57-0.81]^{* * *}$ \\
\hline Females $\geq 40$ years & 20.8 & $5.1(181)$ & $4.8(1)$ & $0.80[0.66-0.98]^{* * *}$ \\
\hline \multicolumn{5}{|l|}{ Marital status: } \\
\hline Married & 55.3 & $4.3(1282)$ & $5.0(7)$ & Reference \\
\hline Not married & 37.9 & $5.6(1158)$ & $9.8(9)$ & $1.35[1.22-1.50]^{* * *}$ \\
\hline Divorced/widowed & 6.8 & $8.7(320)$ & $11.8(4)$ & $2.34[1.97-2.77]^{* * *}$ \\
\hline \multicolumn{5}{|c|}{ Household monthly income (Baht): } \\
\hline$\leq 10000$ & 21.8 & $7.6(962)$ & $9.0(6)$ & $1.91[1.68-2.16]^{* * *}$ \\
\hline $10001-20000$ & 24.3 & $5.0(709)$ & $8.3(6)$ & $1.40[1.20-1.56]^{* * *}$ \\
\hline $20001-30000$ & 21.9 & $4.8(606)$ & $5.6(4)$ & $1.40[1.23-1.61]^{* * *}$ \\
\hline$>30000$ & 31.9 & $3.7(690)$ & $9.7(7)$ & Reference \\
\hline \multicolumn{5}{|l|}{ Paid work: } \\
\hline$\leq 10$ hours per week & 31.4 & $5.9(1062)$ & $4.2(4)$ & Reference \\
\hline 10-40 hours per week & 33.9 & $4.5(870)$ & $5.4(5)$ & 0.90 [0.80-1.01] \\
\hline$>40$ hours per week & 34.8 & $4.9(973)$ & $15.1(13)$ & 1.12 [0.99-1.24] \\
\hline \multicolumn{5}{|l|}{ Residence: } \\
\hline Rural & 44.0 & $5.2(1344)$ & $7.6(10)$ & Reference \\
\hline Urban & 56.0 & $5.1(1653)$ & $8.4(13)$ & 0.99 [0.94-1.06] \\
\hline \multicolumn{5}{|l|}{ Health behaviours } \\
\hline \multicolumn{5}{|l|}{ Smoking: } \\
\hline Never & 76.8 & $4.9(2190)$ & $8.2(14)$ & Reference \\
\hline Current & 8.9 & $7.2(369)$ & $5.2(3)$ & $1.89[1.63-2.19]^{* * *}$ \\
\hline Former & 14.3 & $5.2(434)$ & $8.1(5)$ & $1.22[1.08-1.39]^{* * *}$ \\
\hline \multicolumn{5}{|l|}{ Alcohol drinking: } \\
\hline None per week & 72.9 & $5.0(2078)$ & $8.3(16)$ & \\
\hline 1-2 sessions per week & 19.1 & $4.9(534)$ & $9.1(5)$ & $1.31[1.16-1.48]^{* * *}$ \\
\hline $3+$ sessions per week & 8.0 & $6.3(289)$ & $2.6(1)$ & $1.60[1.36-1.88]^{* * *}$ \\
\hline \multicolumn{5}{|l|}{ Physical activity: } \\
\hline None per week & 22.5 & $5.7(1850)$ & $6.2(9)$ & Reference \\
\hline 1-2 times per week & 33.2 & $5.3(1021)$ & $8.1(6)$ & $0.71[0.63-0.81]^{* * *}$ \\
\hline 3-5 times per week & 32.6 & $4.2(806)$ & $10.2(11)$ & $0.41[0.36-0.47]^{* * *}$ \\
\hline$>5$ times per week & 11.7 & $5.0(341)$ & $8.8(3)$ & $0.37[0.32-0.44]^{* * *}$ \\
\hline \multicolumn{5}{|c|}{ Health and social attributes } \\
\hline \multicolumn{5}{|l|}{ Self-assessed health: } \\
\hline Excellent/very good & 64.6 & $3.0(1159)$ & $4.9(9)$ & Reference \\
\hline Fair & 29.9 & $7.1(1266)$ & $10.3(9)$ & $5.7[5.10-6.33]^{* * *}$ \\
\hline Poor or very poor & 5.4 & $19.4(627)$ & $23.1(6)$ & $19.7[0.42-0.56]^{* * *}$ \\
\hline
\end{tabular}


Table 1 Unhappiness and mortality by cohort attributes, Thai Cohort Study (Continued)

\begin{tabular}{|c|c|c|c|c|}
\hline \multicolumn{5}{|c|}{ Cardio-metabolic conditions: } \\
\hline No & 81.1 & $5.1(2349)$ & $7.5(14)$ & Reference \\
\hline Yes & 18.9 & $5.2(715)$ & $9.3(10)$ & $1.13[1.02-1.26]^{* * *}$ \\
\hline \multicolumn{5}{|l|}{ Social interaction: } \\
\hline Every week & 17.0 & $3.0(306)$ & $1.7(1)$ & Reference \\
\hline 1-2 times/month & 64.8 & $4.5(1645)$ & $8.2(13)$ & $2.12[1.55-2.44]^{* * *}$ \\
\hline Rarely/never & 18.2 & $8.8(903)$ & $15.8(9)$ & $5.02[4.28-5.88]^{* * *}$ \\
\hline
\end{tabular}

*Unhappiness is classified in 2009 as those reporting happy 'little' or 'none of the time'.

**Bivariate odds ratios are adjusted for age-sex categories for all variables and estimate effects of unhappiness.

***Statistical significance at $\mathrm{p}<0.05$.

all, most, some, little, or none of the time). Unhappiness was classified for those reporting being happy 'little' or 'none of the time'. Over the next four years, using the Citizen ID numbers and national vital statistics, we detected 296 deaths among the 60569 cohort members studied in 2009. This 4-year longitudinal analysis focused on unhappiness and its association with mortality. Covariates investigated include sociodemographic characteristics (age-sex categories, age in years, marital status, household monthly income, paid work hours, rural-urban residence), health behaviours (smoking, alcohol drinking, physical activity), health states (self-assessed overall health and doctor diagnosed cardio-metabolic conditions), and social capital (interaction with family, friends, neighbours, and colleagues). These covariates were also controlled for in a recent systematic review of 70 quantitative studies on positive wellbeing and mortality [7].

For our study, using the 2009 cohort follow-up data, we first performed a bivariate analysis of the crosssectional relationship between covariates and unhappiness (Table 1). Then we conducted a 4-year longitudinal analysis of 2009 unhappiness as a predictor of mortality for the 296 deaths occurring in the period over 20092012 (Table 2). For this analysis of the effect of unhappiness on mortality, the odds ratios for each variable in the model are adjusted for the influence for all the covariates listed in Table 1. These Adjusted Odds Ratios (AOR) are presented together with their 95\% Confidence Intervals in Table 2.

Table 1 presents a breakdown of cohort attributes by unhappiness and mortality status. Approximately 4-5\% of cohort members reported being unhappy in 2009 across age-sex categories among those who remained alive four years later. The corresponding percentages were much higher among those who died (4.7-10.7\%). Age-sex adjusted bivariate associations linking cohort attributes to unhappiness are also reported in Table 1. Compared to those married, cohort members who were not married or divorced/widowed were 1.35 and 2.34 times more likely to report being unhappy. Former smokers and current smokers were more likely to report being unhappy compared to non-smokers (age-sex adjusted odds ratios 1.22 and 1.89). Alcohol drinking was associated with unhappiness and physical activity was a statistically significant protective factor. Unhappiness was also associated with low social interaction and with self-assessed 'poor' or 'very poor' health.

Cohort members who reported being happy 'little' or 'none of the time' in 2009 were more likely to die in the subsequent four years (Adjusted Odds Ratio - AOR 2.60, 95\% CI 1.17-5.80). Other significant covariates include being female $(<40$ years AOR $0.66, \geq 40$ years AOR 0.57), unmarried (AOR 1.64) and current smokers (AOR 2.45).

Previous reports from Finland suggest possible pathways between unhappiness and mortality. One route is via unintentional injury (hazard ratio 2.83 over 15 years) mediated through adverse health behaviors [8]; an alternative route involves intentional injury (hazard ratio 7.01 over 20 years) through self-harm and suicide [12]. Another report using German socio-economic panel data found that a $10 \%$ increase in happiness associated with $4 \%$ decrease in probability of dying. The results were more pronounced among males, those unmarried, and the chronically ill [13]. For our Thai Cohort Study, after adjusting for covariates, we also found males and those who never married in 2009 were significantly more likely to die over the next four years. Initial health states (self-assessed overall health and cardio-metabolic conditions) were in the model but they were not statistically significant in the multivariate analyses.

We also investigate how self-reported happiness connects to other standard measures within our cohort. First, in our earlier paper drawing from the same 2009 cohort data, we noted that self-reported happiness correlated with standard measures of life satisfaction [16]. Second, a 2005-2009 longitudinal analysis of low life satisfaction in the cohort (data not shown) confirms that cohort participants who reported being unhappy were much more likely to report low life satisfaction not only in 2009 but also four years earlier (2005). This 
Table 2 Self-reported happiness as a predictor of mortality, Thai Cohort Study 2009-2012

\begin{tabular}{lc}
\hline Cohort attributes & $\begin{array}{c}\text { Multivariate adjusted odds ratio* }[95 \% \\
\text { confidence interval] of mortality predictor }\end{array}$ \\
\hline
\end{tabular}

Self-reported happiness**

All of the time

Most of the time

Some of the time

Little of the time

Sociodemographic attributes

Age-sex groups:

Males $<40$ years

Females $<40$ years

Males $\geq 40$ years

Females $\geq 40$ years

Marital status:

Married

Not married

Divorced/widowed

Household monthly income (Baht):

$\leq 10000$

10001-20000

20001-30000

$>30000$

\section{Residence:}

Rural

Urban

Paid work:

$\leq 10$ hours per week

10-40 hours per week

>40 hours per week

Health-risk attributes

Smoking:

Never

Current

Former

Alcohol drinking:

None per week

1-2 sessions per week

$3+$ sessions per week

Physical activities:

None per week

1-2 times per week

3-5 times per week

$>5$ times per week
$1.19[0.41-3.50]$

0.72 [0.43-1.20]

Reference

1.66 [0.88-3.11]

1.69 [0.88-3.23]

$2.60[1.17-5.80]^{* * *}$

Reference

$\mathbf{0 . 6 6}[0.43-0.99]^{* * *}$

1.34 [0.78-2.31]

$\mathbf{0 . 5 7}[0.21-0.74]^{* * *}$

Reference

$1.67[1.17-2.39]$ ***

0.99 [0.24-4.16]

1.45 [0.71-2.98]

1.44 [0.84-2.49]

Reference

Reference

0.89 [0.66-1.20]

Reference

0.83 [0.51-1.35]

0.74 [0.34-1.61]

Reference

$2.45[1.61-3.74]^{* * *}$

$1.09[0.71-1.66]$

$1.27[0.42-3.90]$

Reference

0.68 [0.45-1.03]

$0.96[0.65-1.44]$

$0.72[0.39-1.32]$
Table 2 Self-reported happiness as a predictor of mortality, Thai Cohort Study 2009-2012 (Continued)

Health and social

attributes

Self-assessed health:

Excellent/very good

Reference

Fair

0.97 [0.70-1.34]

Poor or very poor

$1.31[0.74-2.31]$

Cardio-metabolic

conditions:

No

Reference

Yes

1.33 [0.97-1.84]

Social interaction:

Every week Reference

$1-2$ times/month $\quad 1.08$ [0.57-2.05]

Rarely/never $\quad 1.69$ [0.62-4.58]

*The Odds Ratio for each variable is mutually adjusted for the influence of all the other variables in the Table.

**The question asks "in the past four weeks, how much of the time did you feel happy?".

***Statistical significance at $p<0.05$.

link to low life satisfaction is important as several studies have shown that low life satisfaction is on the pathway to all-cause mortality $[7-9,17,18]$. These several strands of evidence collectively indicate that our happiness data have construct and content validity, connecting well to previous literature.

A general relationship between happiness and mortality might seem obvious but there is almost no actual evidence that this is so beyond several reports from affluent economies. Our study provides longitudinal evidence from Thailand and thus is one of the first from a non-affluent region. Our study was prospective and we controlled for a wide array of potential covariates including age, sex, income, behaviours, and health states. Additional variables such as doctor visits were used in other happinessmortality studies [13] but were not available in our data. Some residual confounding is possible but we note that most of the variables that have been shown to relate to all-cause mortality are in our model. We also acknowledge the limitation of the short mortality follow-up (4 years) and future longitudinal analyses will confirm if the findings hold in the longer term. Even with the small number of deaths investigated, we were able to capture an epidemiologically and statistically significant relationship between self-reported unhappiness and subsequent death.

We conclude that happiness is not just a factor linked to longevity in affluent countries, but also increases the probability of staying alive in a middle-income Asian setting. It would be helpful if this report stimulates studies of happiness and mortality elsewhere in the world. So far it appears the happiness effect noted here is indeed 
independent of wealth and culture and we expect that this can be found in other settings. The implications are that the pursuit of happiness is likely to add to life expectancy and is a legitimate strategy to improve population health.

\section{Ethical approval}

Informed written consent was obtained from all participants. All students were advised that they could withdraw, or not participate, without any effect on their academic progress. The questionnaires never sought sensitive personal information and no biological samples were taken. Ethics approval was obtained from Sukhothai Thammathirat Open University Research and Development Institute (protocol 0522/10) and the Australian National University Human Research Ethics Committee (protocols 2004/344 and 2009/570).

\section{Abbreviations}

AOR: Adjusted odds ratio; 95\% Cl: 95\% confidence interval; ID: Identity.

\section{Competing interests}

The authors declare that they have no competing interest.

\section{Authors' contributions}

V conceptualised the study, analysed data, and wrote a draft manuscript. AS provided comments on original manuscript and revision. AS and SS led the Thai Cohort Study. All authors read and approved the final manuscript.

\section{Acknowledgments}

We thank the staff at Sukhothai Thammathirat Open University (STOU) who assisted with student contact and the STOU students who are participating in the cohort study. We also thank Dr. Bandit Thinkamrop and his team from Khon Kaen University for support on initial data processing. As well we would like thank the Thai Ministry of Interior for mortality investigation. Matthew Kelly and Peter Sbirakos provided editorial assistance.

\section{Funding}

This study was supported by the International Collaborative Research Grants Scheme with joint grants from the Wellcome Trust UK (GR071587MA) and the Australian National Health and Medical Research Council (268055), and as a global health grant from the NHMRC (585426).

\section{Author details}

${ }^{1}$ National Centre for Epidemiology and Population Health, College of Medicine, Biology and Environment, Research School of Population Health, The Australian National University, Building 62, Mills Rd, Canberra, Acton 2601, Australia. ${ }^{2}$ School of Human Ecology, Sukhothai Thammathirat Open University, Nonthaburi, Thailand.

Received: 3 April 2014 Accepted: 23 July 2014

Published: 7 August 2014

\section{References}

1. Tobgay T, Dophu U, Torres CE, Na-Bangchang K: Health and Gross National Happiness: review of current status in Bhutan. J Multidiscip Healthc 2011, 4:293-298.

2. Bok S: Exploring Happiness: From Aristotle to Brain Science. New Haven: Yale University Press; 2010.

3. Michalos AC: Encyclopedia of quality of life and well-being research. Dordrecht, Netherlands: Springer; 2014.

4. Delle Fave A, Brdar I, Freire T, Vella-Brodrick D, Wissing M: The eudaimonic and hedonic components of happiness: qualitative and quantitative findings. Soc Indic Res 2011, 100:185-207.

5. Sabatini F: The relationship between happiness and health: Evidence from Italy. Soc Sci Med 2014, 114:178-187.
6. Siahpush M, Spittal M, Singh GK: Happiness and life satisfaction prospectively predict self-rated health, physical health, and the presence of limiting, long-term health conditions. Am J Health Promot 2008, 23(1):18-26.

7. Chida Y, Steptoe A: Positive psychological well-being and mortality: a quantitative review of prospective observational studies. Psychosom Med 2008, 70(7):741-756

8. Koivumaa-Honkanen H, Honkanen R, Koskenvuo M, Viinamaki H, Kaprio J: Life dissatisfaction as a predictor of fatal injury in a 20-year follow-up. Acta Psychiatr Scand 2002, 105(6):444-450.

9. Bray I, Gunnell D: Suicide rates, life satisfaction and happiness as markers for population mental health. Soc Psychiatry Psychiatr Epidemiol 2006, 41(5):333-337.

10. Ryan RM, Deci EL: On happiness and human potentials: a review of research on hedonic and eudaimonic well-being. Annu Rev Psychol 2001, 52:141-166.

11. Inglehart R, Foa R, Peterson C, Welzel C: Development, freedom, and rising happiness: A global perspective (1981-2007). Perspect Psychol Sci 2008, 3:264-285.

12. Koivumaa-Honkanen $H$, Honkanen R, Koskenvuo M, Kaprio J: Self-reported happiness in life and suicide in ensuing 20 years. Soc Psychiatry Psychiatr Epidemiol 2003, 38(5):244-248.

13. Guven C, Saloumidis R: Why is the world getting older? The influence of happiness on mortality. In Deutsches Institut für Wirtschaftsforschung. Berlin: German Socio-Economic Panel Study (SOEP) papers on Multidisciplinary Panel Data Research; 2009.

14. Sleigh AC, Seubsman SA, Bain C: Cohort profile: The Thai Cohort of 87 134 Open University students. Int J Epidemiol 2008, 37(2):266-272.

15. Seubsman SA, Yiengprugsawan V, Sleigh AC, Thai Cohort Study T: A large national Thai Cohort Study of the Health-Risk Transition based on Sukhothai Thammathirat Open University students. ASEAN J Open Distance Learn 2012, 4(1):58-69.

16. Yiengprugsawan V, Somboonsook B, Seubsman S, Sleigh AC: Happiness, mental health, and socio-demographic associations among a national cohort of Thai adults. J Happiness Stud 2012, 13(6):1019-1029.

17. Collins AL, Glei DA, Goldman N: The role of life satisfaction and depressive symptoms in all-cause mortality. Psychol Aging 2009, 24(3):696-702

18. Iwasa H, Kawaai C, Gondo Y, Inagaki H, Suzuki T: [Subjective well-being and all-cause mortality among middle-aged and elderly people living in an urban Japanese community]. Nihon Ronen Igakkai Zasshi 2005, 42(6):677-683.

doi:10.1186/1751-0759-8-18

Cite this article as: Yiengprugsawan et al:: Unhappiness and mortality: evidence from a middle-income Southeast Asian setting. BioPsychoSocial Medicine 2014 8:18.

\section{Submit your next manuscript to BioMed Central and take full advantage of:}

- Convenient online submission

- Thorough peer review

- No space constraints or color figure charges

- Immediate publication on acceptance

- Inclusion in PubMed, CAS, Scopus and Google Scholar

- Research which is freely available for redistribution 